\title{
MAC Games for Distributed Wireless Network Security with Incomplete Information of Selfish and Malicious User Types
}

\author{
Yalin Evren Sagduyu, Randall Berry and Anthony Ephremides
}

\begin{abstract}
We consider game theoretic models of wireless medium access control (MAC) in which each transmitter makes individual decisions regarding their power level or transmission probability. This allows for scalable distributed operation; however, it can also enable users to pursue malicious objectives such as jamming other nodes to deny them service. We study games with two types of players: selfish and malicious transmitters. Each type is characterized by a utility function depending on throughput reward and energy cost. Furthermore, we focus on the setting where the transmitters have incomplete information regarding other transmitters' types, modeled as probabilistic beliefs. We first analyze a power-controlled MAC game in which the nodes select powers for continuous transmissions and then extend this to a random access MAC in which nodes choose transmission probabilities. For each case, the Bayesian Nash equilibrium strategies are derived for different degrees of uncertainty, and the resulting equilibrium throughput of selfish nodes is characterized. We identify conditions in which the throughput improves with increasing type uncertainty and introduce Bayesian learning mechanisms to update the type beliefs in repeated games. For unknown types and costs, we also specify the equilibrium cut-off thresholds for monotonic transmission decisions. The analysis provides insights into the optimal defense mechanisms against denial of service attacks at the MAC layer in wireless networks.
\end{abstract}

Index Terms-Bayesian games; distributed operation; incomplete information; malicious users; MAC; power control; random access; security; selfish users.

\section{INTRODUCTION}

In wireless networks, centralized access control does not scale with the number of nodes. A promising alternative is distributed control in which nodes independently select their transmission strategies to optimize individual performance objectives. Such systems are naturally modeled as non-cooperative games, e.g., [1]-[3] consider power control games and [4]-[6] consider random access games for singlereceiver access control. We consider models for both of these situations as well.

Non-cooperative nodes may pursue not only selfish but also malicious objectives such as interfering with the packet transmissions of other nodes. In this context, the channel jamming effects of malicious transmitters have been evaluated in terms of the worst-case throughput performance for ALOHA systems in [7] and have been incorporated into a zero-sum game of balancing the mutual information over

Y. E. Sagduyu and R. Berry are with the Department of Electrical Engineering and Computer Science, Northwestern University, Evanston, IL 60208, USA. y-sagduyu@northwestern.edu, rberrydece.northwestern.edu

A. Ephremides is with the Department of Electrical and Computer Engineering, University of Maryland, College Park, MD 20742, USA. etony@umd.edu
Gaussian channels in [8]. For both models of power control and random access, jamming games have been formulated for users with known selfish or malicious types reflected in their utilities [9]-[11]. The possible misbehavior of transmitters has been studied in [12]-[13] for intrusion detection and in [14] for packet forwarding without MAC interactions.

In this paper, we strip off all the complexities introduced by multihop operation and analyze the fundamental interactions of selfish and malicious nodes at the MAC layer. This problem has been studied in [9]-[10] under power control and random access for the case of known types of selfish and malicious nodes (with known utility functions). However, the Nash equilibrium strategies strongly depend on the (selfish or malicious) user types. In practice, malicious nodes would likely conceal their intent, i.e., nodes would have incomplete information regarding the types of other nodes. Here, we study the effects of such incomplete information.

Our goal is to develop a framework for denial of service attacks as dynamic non-cooperative games among selfish nodes transmitting to a common receiver and malicious nodes jamming their transmissions. Although our primary focus is on the case of two transmitters, we also extend the analysis to an arbitrary number of selfish and malicious transmitters. The node types are possibly hidden and represented by probabilistic beliefs at individual nodes. The performance objectives incorporate (i) throughput rewards (based on Signalto-Interference-plus-Noise-Ratio (SINR) for power control or success probability for random access), (ii) transmission energy costs, and (iii) malicious jamming interests.

Malicious nodes do not have any incentive of jamming each other's transmissions and they become less aggressive in their attack decisions, if they are uncertain about whether the opponent nodes are selfish. This suggests a distributed security paradigm that allows random malicious node behavior (without external detection) and relies on individual nodes to hide their types as an inherent defense mechanism to mitigate the malicious operation. We evaluate the Bayesian Nash equilibrium strategies and present the conditions in which the type uncertainty may be beneficial for selfish nodes to improve their throughput properties.

For random access, we also derive the equilibrium set of thresholds for the monotonic transmission decisions under the incomplete information on types and cost parameters. A similar problem has been considered in [15] for selfish nodes with known types only. Instead, we formulate the uncertainty of node types and (energy) costs jointly.

In addition to one-stage Bayesian games, we extended the results to consider dynamic repeated games. The usual 
approach to intrusion detection is based on externally detecting malicious behavior, e.g., by hypothesis testing, if the transmission strategies of selfish and malicious nodes are fixed and known [16]. Instead, we introduce a distributed solution to the network security problem at the MAC layer in which nodes learn each other's types according to a Bayesian learning mechanism and play the best-response strategies against their type beliefs that evolve dynamically over time.

The paper is organized as follows. Section II introduces the game model for power-controlled MAC with two users. The interactions of possibly selfish or malicious users are presented in Sections III and IV for two different SINR-based throughput reward functions. This is followed in Section V by the analysis of Bayesian learning mechanisms for selfish and malicious user types with unknown belief distributions. We outline the generalization to an arbitrary number of users in Section VI. The game model is extended in Section VII to consider probabilistic transmission decisions for random access. Finally, we draw conclusions and present thoughts for future work in Section VIII.

\section{Two-User Power-Controlled MAC GAme}

Consider a one-stage game between two transmitters of two possible (selfish or malicious) types. Define $P_{i} \geq 0$ and $E_{i} \geq 0$ as the transmission power and the corresponding energy cost (per unit power) of node $i=1,2$, respectively. Assume a synchronous slotted system, in which each packet transmission takes one time slot. Each node $i=1,2$ independently chooses the power $P_{i}$ for transmitting to a common receiver in order to maximize the individual expected utility $u_{i}\left(P_{1}, P_{2}\right)$. The SINR value achievable by selfish node $i$ is

$$
\gamma_{i}=\frac{h_{i} P_{i}}{\frac{1}{L} \sum_{j \neq i} h_{j} P_{j}+\sigma^{2}},
$$

where $h_{i}$ is the channel gain for node $i, L$ is the processing gain and $\sigma^{2}$ is the channel noise. The (throughput) reward for a selfish node $i$ is $f_{i}\left(\gamma_{i}\right)$, which is an increasing function of the SINR value $\gamma_{i}$. We consider two different reward functions for selfish nodes:

$$
f_{i}\left(\gamma_{i}\right)=\gamma_{i}, \quad f_{i}\left(\gamma_{i}\right)=\log \left(1+\gamma_{i}\right),
$$

namely, the SINR value $\gamma_{i}$ and the Shannon rate from transmitter $i$ to the receiver for the SINR value $\gamma_{i}$.

Any selfish node $i$ also incurs the energy cost $E_{i} P_{i}$ proportional to power $P_{i}$ and maximizes the expected utility

$$
u_{i}\left(P_{1}, P_{2}\right)=f_{i}\left(\gamma_{i}\right)-E_{i} P_{i} .
$$

Any malicious node $i=1,2$ incurs as cost the throughput reward $f_{j}\left(\gamma_{j}\right)$ of selfish opponent $j \neq i$ as well as the energy cost $E_{i} P_{i}$ and so maximizes the expected utility

$u_{i}\left(P_{1}, P_{2}\right)=\left\{\begin{array}{c}-f_{j}\left(\gamma_{j}\right)-E_{i} P_{i}, \\ \text { if the opponent node } j \text { is selfish, } \\ -E_{i} P_{i}, \\ \text { if the opponent node } j \text { is malicious, }\end{array}\right.$

for $j=1,2, j \neq i$, i.e., malicious nodes do not have any incentive of interfering with each other's transmissions. Note that without energy costs, a game between one malicious node and one selfish node will be a zero-sum game of throughput balancing. ${ }^{1}$ However, we end up with a non-zerosum game for the case of non-zero energy costs.

Let $\phi_{i}$ denote the probabilistic belief of node $i$ that the other node $j \neq i$ is selfish. The parameters $L, \sigma^{2}, h_{i}, E_{i}$ and $\phi_{i}, i=1,2$, are known to all nodes. This models the case when the system is monitored and nodes are notified with the long-term statistics of attack possibilities. We will consider the effects of uncertainty on type belief distributions and energy costs in Sections V and VII.

\section{INTERACTIONS OF SELFISH AND MALICIOUS TRANSMITTERS FOR REWARD FUNCTION $f_{i}\left(\gamma_{i}\right)=\gamma_{i}$}

We next present results for two transmitters with reward function $f_{i}\left(\gamma_{i}\right)=\gamma_{i}$, under different assumptions on the type uncertainty. In general, the Nash equilibrium strategies $P_{i}^{*}$ for any node $i$ satisfy

$$
u_{i}\left(P_{i}^{*}, P_{-i}^{*}\right) \geq u_{i}\left(P_{i}, P_{-i}^{*}\right), \quad i=1,2,
$$

for any strategy $P_{i}, i=1,2$, where $P_{-i}$ is the strategy of the node(s) other than node $i$, such that no node can unilaterally improve its individual performance beyond the Nash equilibrium.

\section{A. Known Types of Two Transmitters}

Theorem 1: For two selfish transmitters, the unique Nash equilibrium strategies are $^{2}$ :

$$
\begin{array}{r}
P_{i}=\frac{L}{h_{i}}\left(\frac{h_{j}}{E_{j}}-\sigma^{2}\right), j \neq i, \quad \text { if } h_{i} \geq \sigma^{2} E_{i}, i=1,2, \\
P_{i}=0, \text { if } h_{i}<\sigma^{2} E_{i}, i=1,2, \\
P_{i}=0, P_{j} \rightarrow \infty, \quad \text { if } h_{i}<\sigma^{2} E_{i}, h_{j}>\sigma^{2} E_{j}, j \neq i .
\end{array}
$$

Proof: The individual optimization problem for any transmitter $i=1,2$ is given by

$$
\max _{P_{i} \geq 0} u_{i}\left(P_{i}, P_{-i}\right), \text { for } P_{-i} \geq 0 .
$$

For each user, define the Lagrangian

$$
L_{i}\left(P_{1}, P_{2}\right)=u_{i}\left(P_{1}, P_{2}\right)+\lambda_{i} P_{i}, \quad i=1,2,
$$

where $\lambda_{i} \geq 0$ is a Lagrange multiplier corresponding to the inequality constraint in (9). The Karush-Kuhn-Tucker (KKT) conditions for the optimal solutions of (9) are given by

$$
\frac{\partial L_{i}\left(P_{1}, P_{2}\right)}{\partial P_{i}}=0, P_{i} \geq 0, \lambda_{i} \geq 0, \lambda_{i} P_{i}=0, i=1,2 .
$$

These necessary conditions are also sufficient for optimality, since the utility $u_{i}\left(P_{1}, P_{2}\right)$ and inequality constraint $P_{i} \geq$ 0 are continuously differentiable and concave functions of $P_{i}$. The equilibrium strategies (6)-(8) follow from applying the KKT conditions (11) separately to each objective function

\footnotetext{
${ }^{1}$ We could also define the malicious utility by using a reward function $\mathcal{K}-f_{j}\left(\gamma_{j}\right)$ to be maximized (for a constant $\mathcal{K}$ ). However, this would not change the equilibrium strategies of selfish or malicious nodes.

${ }^{2}$ Similar utility functions have been considered in [1]-[3] for selfish nodes with throughput and energy efficiency objectives.
} 
$u_{i}\left(P_{1}, P_{2}\right), i=1,2$, with constraint $P_{i} \geq 0$, where the utilities $u_{i}\left(P_{1}, P_{2}\right), i=1,2$, are given by (3).

For a power-controlled MAC, the performance measure of interest is the SINR value achievable by selfish nodes. The SINR value achievable by selfish node $i=1,2$ in Nash equilibrium is given by

$$
\gamma_{i}=\left\{\begin{array}{cc}
E_{i} P_{i}, & \text { if } h_{j}>\sigma^{2} E_{j}, \quad j=1,2, j \neq i . \\
\frac{h_{i} P_{i}}{\sigma^{2}}, & \text { otherwise, }
\end{array}\right.
$$

Theorem 2: For selfish transmitter 1 and malicious transmitter 2, the unique Nash equilibrium strategies are

$$
\begin{array}{r}
P_{1}=\frac{L}{h_{2}} \frac{E_{2} h_{1}}{\left(E_{1}\right)^{2}}, P_{2}=\frac{L}{h_{2}}\left(\frac{h_{1}}{E_{1}}-\sigma^{2}\right), \text { if } h_{1} \geq \sigma^{2} E_{1}, \\
P_{1}=0, P_{2}=0, \text { if } h_{1}<\sigma^{2} E_{1} .
\end{array}
$$

Proof: The equilibrium strategies (13)-(14) follow from applying the KKT conditions (11) separately to each objective function $u_{i}\left(P_{1}, P_{2}\right), i=1,2$, with constraint $P_{i} \geq 0$, where the utilities $u_{1}\left(P_{1}, P_{2}\right)$ and $u_{2}\left(P_{1}, P_{2}\right)$ are given by (3) and (4), respectively.

The equilibrium SINR of selfish node 1 is given by (12) with $P_{1}$ from (13)-(14). The malicious attack of node 2 is more successful in reducing the SINR of selfish node 1 compared to the alternative selfish behavior of node 2 (under the assumption of $h_{i} \geq \sigma^{2} E_{i}, i=1,2$, for the non-zero transmission powers), if and only if $\frac{h_{2}}{E_{2}}>\sigma^{2}+\frac{E_{2}}{h_{2}}\left(\frac{h_{1}}{E_{1}}\right)^{2}$, i.e., if $h_{1}$ is small and $E_{1}$ is large. Otherwise, we observe the windfall of malice, i.e., the malicious attack fails compared to the selfish operation (as noted before for the separate problem of routing [17]). If both transmitters are malicious, they do not receive any reward from interfering with each other and the Nash equilibrium strategies are $P_{1}=0$ and $P_{2}=0$.

The system parameters may not be perfectly known or may be random. Then, any jamming node randomly chooses to pursue either selfish or malicious objective functions. Therefore, each node would face an opponent of random identity with the degree of uncertainty depending on the distributions of system parameters.

\section{B. Selfish Transmitter 1 (Known Type) and Transmitter 2 of Unknown Type}

Assume now that selfish node 1 believes that node 2 is selfish with probability $\phi_{1}$ (known to node 2). Define $P_{2, S}$ and $P_{2, M}$ as the power, $E_{2, S}$ and $E_{2, M}$ as the energy cost, and $h_{2, S}$ and $h_{2, M}$ as the channel gain for selfish and malicious node 2 , respectively.

Theorem 3: For selfish transmitter 1 and transmitter 2 of unknown type, the Bayesian Nash equilibrium strategies for $\phi_{1} \in(0,1)$ are

$$
\begin{aligned}
P_{1} & =\frac{L}{h_{1}}\left(\frac{h_{2, S}}{E_{2, S}}-\sigma^{2}\right), \\
P_{2, S} & =\frac{L}{h_{2, S}}\left[\frac{h_{1} \phi_{1}}{\left(E_{1}-\frac{\left(1-\phi_{1}\right) h_{1}}{\frac{h_{2, M}}{L} P_{2, M}+\sigma^{2}}\right)}-\sigma^{2}\right]^{+}, \\
P_{2, M} & =\left[\sqrt{\frac{L h_{1} P_{1}}{h_{2, M} E_{2, M}}-\frac{L \sigma^{2}}{h_{2, M}}}\right]^{+},
\end{aligned}
$$

if $h_{2, S} \geq \sigma^{2} E_{2, S}$, where $[x]^{+}=\max (x, 0)$. Otherwise,

$$
P_{1}=\left(\left[E_{1}-\frac{\phi_{1} h_{1}}{\sigma^{2}}\right]^{+}\right)^{-2} \frac{E_{2, M} L h_{1}}{h_{2, M}}\left(1-\phi_{1}\right)^{2}
$$

$P_{2, S}=0$ and $P_{2, M}$ is given by (17) with $P_{1}$ from (18).

Proof: The equilibrium strategies (16)-(18) follow from applying the KKT conditions separately to the objective functions $u_{2, S}\left(P_{1}, P_{2, S}\right)$ of selfish node 2 and $u_{2, M}\left(P_{1}, P_{2, M}\right)$ of malicious node 2 with constraints $P_{1} \geq$ $0, P_{2, S} \geq 0$ and $P_{2, M} \geq 0$, where the utilities $u_{2, S}$ and $u_{2, M}$ are given by (3) and (4), respectively. Then, the equilibrium strategy (15) follows from applying the KKT conditions (11) to the expected objective function $\bar{u}_{1}\left(P_{1}, P_{2, S}, P_{2, M}\right)=$ $\phi_{1} u_{1}\left(P_{1}, P_{2, S}\right)+\left(1-\phi_{1}\right) u_{1}\left(P_{1}, P_{2, M}\right)$ of selfish node 1 , where the utility $u_{1}$ is given by (3).

Assume $h_{2, S} \geq \sigma^{2} E_{2, S}$ for the case of non-zero transmission power of selfish node 2 . Selfish node 1 can individually detect the type of node 2, unless $P_{2, S}=P_{2, M}$ in (16)-(17). If node 1 detects the other node as malicious such that $\phi_{1}=0$, the power $P_{1}$ is changed to (13), whereas the power of node 2 is still given by (17).

Consider the case when node 2 is selfish. In Nash equilibrium, $P_{1}$ is the same as in the case of two selfish nodes with known types (independent of $\phi_{1}$ ). So, selfish node 2 cannot learn, whether node 1 detects the type of node 2 , or not, and continues to operate with the same value of $\phi_{1}$ as before. The uncertainty of the opponent's type is beneficial for selfish node 2 (i.e., selfish node 2 can increase $\gamma_{2, S}$ by hiding its type compared to the case with the known types), if $\frac{h_{1}}{E_{1}}>\left(\frac{h_{2, M}}{L} P_{2, M}+\sigma^{2}\right)$, where $P_{2, M}$ follows from (17), i.e., if $h_{1}$ is large and $E_{1}$ is small. Otherwise, selfish node 2 should reveal its type.

On the other hand, the uncertainty on the opponent's type is beneficial for selfish node 1 , if $\frac{h_{1}}{E_{1}}<\left(\frac{h_{2, M}}{L} P_{2, M}+\sigma^{2}\right)$, i.e., if $h_{1}$ is small and $E_{1}$ is large.

Next, consider the case when node 2 is malicious. Then, the power $P_{2, M}$ does not depend on $\phi_{1}$. The attack of node 2 is more successful (in reducing the SINR value $\gamma_{1}$ ) by hiding its type (compared to the case of known types of selfish node 1 and malicious node 2), if and only if $\frac{E_{2, M}}{h_{2, M}}\left(\frac{h_{1}}{E_{1}}\right)^{2}>\frac{h_{2, S}}{E_{2, S}}-$ $\sigma^{2}$ (i.e., if $h_{1}$ is large and $E_{1}$ is small). As a result, selfish and malicious nodes choose between revealing and concealing their identities depending on the system parameters.

The equilibrium SINR value achievable by selfish nodes is depicted in Figure 1 as function of the type belief probability $\phi_{1}$, where the expected SINR $\gamma_{1}$ of selfish node 1 is averaged over the type of node 2 under the assumption that the type belief distribution $\phi_{1}$ is equal to the true distribution $\tilde{\phi}$ that node 2 is selfish. In Figure 2, we illustrate the case when the type belief distribution $\phi_{1}$ deviates from the true distribution $\tilde{\phi}$. Note that assuming the true distribution $\tilde{\phi}$ for the type belief $\phi_{1}$ does not necessarily optimize the equilibrium SINR value $\gamma_{1}$ of selfish node 1 , i.e., the uncertainty on type belief distributions may possibly improve the equilibrium SINR $\gamma_{1}$. 


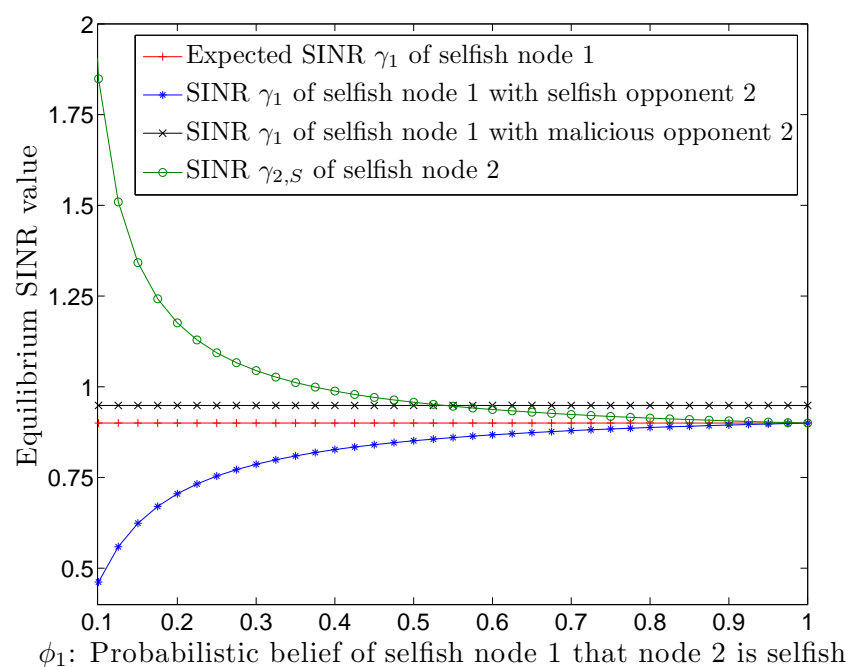

Fig. 1. The equilibrium SINR values of selfish nodes as function of type belief probability $\phi_{1}$ for $h_{i}=1, E_{i}=1, i=1,2, L=1$ and $\sigma^{2}=0.1$.

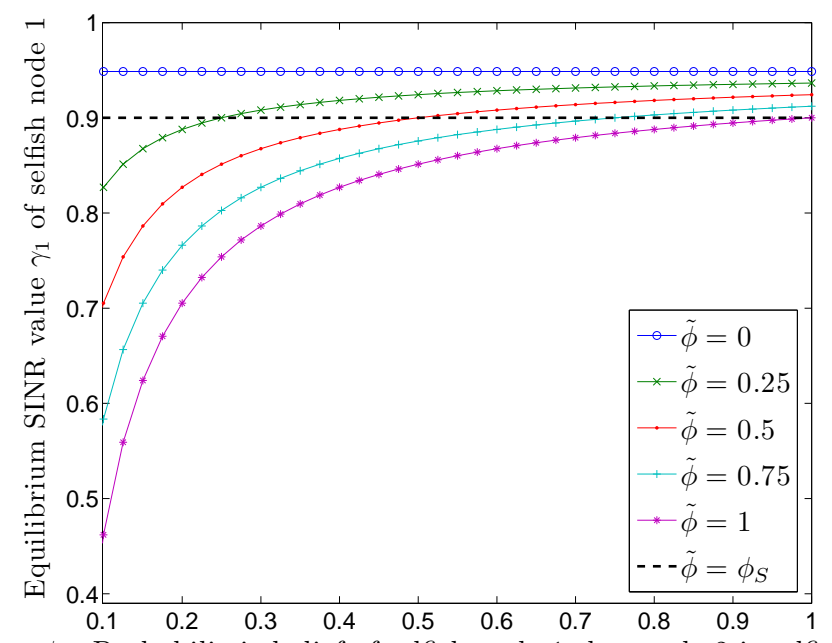

$\phi_{1}$ : Probabilistic belief of selfish node 1 that node 2 is selfish

Fig. 2. The equilibrium SINR value of selfish node 1 as function of type belief probability $\phi_{1}$ for different values of true distribution $\tilde{\phi}$ that node 2 is selfish and for $h_{i}=1, E_{i}=1, i=1,2, L=1$ and $\sigma^{2}=0.1$.

\section{Malicious Transmitter 1 (Known Type) and Transmitter 2 of Unknown Type}

Now assume that malicious node 1 believes that node 2 is selfish with probability $\phi_{1}$ (known to node 2 ).

Theorem 4: For $\phi_{1} \in(0,1)$, the Bayesian Nash equilibrium strategies are

$$
\begin{aligned}
P_{1} & =\frac{L}{h_{1}}\left(\frac{h_{2, S}}{E_{2, S}}-\sigma^{2}\right), \\
P_{2, S} & =\frac{L}{h_{1}} \frac{E_{1} h_{2, S}}{\left(E_{2, S}\right)^{2}} \frac{1}{\phi_{1}}, \\
P_{2, M} & =0,
\end{aligned}
$$

if $h_{2, S} \geq \sigma^{2} E_{2, S}$. Otherwise, $P_{1}=0, P_{2, S}=0, P_{2, M}=0$.
Proof: The equilibrium strategies (20)-(21) follow from applying the KKT conditions (11) separately to the objective functions $u_{2, S}\left(P_{1}, P_{2, S}\right)$ of selfish node 2 and $u_{2, M}\left(P_{1}, P_{2, M}\right)$ of malicious node 2 , with constraints $P_{1} \geq$ $0, P_{2, S} \geq 0$ and $P_{2, M} \geq 0$, where $u_{2, S}$ and $u_{2, M}$ are given by (3) and (4), respectively. The equilibrium strategy (19) follows from applying the KKT conditions (11) to the expected objective function $\bar{u}_{1}\left(P_{1}, P_{2, S}, P_{2, M}\right)=$ $\phi_{1} u_{1}\left(P_{1}, P_{2, S}\right)+\left(1-\phi_{1}\right) u_{1}\left(P_{1}, P_{2, M}\right)$ of malicious node 1 with $u_{1}\left(P_{1}, P_{2, M}\right)=0$ and $u_{1}\left(P_{1}, P_{2, S}\right)$ from (4).

After playing one stage of the game, malicious node 1 would immediately learn the opponent's type by observing the interference power (through the SINR feedback), since $P_{2, S} \neq P_{2, M}$. For $h_{2, S}>\sigma^{2} E_{2, S}$, the equilibrium SINR of selfish node 2 is given by

$$
\gamma_{2, S}=\frac{E_{1} h_{2, S} L}{E_{2, S} h_{1}} \frac{1}{\phi_{1}},
$$

which increases, as $\phi_{1}$ decreases, i.e., a malicious attack becomes less successful (in reducing the equilibrium SINR $\gamma_{2, S}$ of the possibly selfish node 2), as the uncertainty on the opponent's type increases. Therefore, node 2 should hide its type to increase $\gamma_{2, S}$, if it is selfish.

\section{Transmitters 1 and 2 of Unknown Types}

Nodes 1 and 2 have unknown selfish or malicious types. For each selfish and malicious node, define $E_{S}$ and $E_{M}$ as the energy cost, $h_{S}$ and $h_{M}$ as the channel gain, $\phi_{S}$ and $\phi_{M}$ as the probabilistic belief that the opponent is selfish. These parameters are known to both nodes. In this paper, symmetric strategies are considered for identical nodes with common type belief distributions, costs and system parameters. Then, each node assumes that all nodes of the same type choose the same transmission strategy. Define $P_{S}$ and $P_{M}$ as the transmission power of selfish and malicious node, respectively.

Theorem 5: For $\phi_{S} \in(0,1)$ and $\phi_{M} \in(0,1)$, the symmetric Bayesian Nash equilibrium strategies $P_{S}$ and $P_{M}$ of selfish and malicious transmitters are

$$
\begin{aligned}
P_{S} & =\left[P_{S}^{*}\right]^{+}, \\
P_{M} & =\frac{L}{h_{M}}\left[\sqrt{P_{S}} \sqrt{\frac{\phi_{M} h_{S} h_{M}}{L E_{M}}}-\sigma^{2}\right]^{+},
\end{aligned}
$$

respectively, where $P_{S}^{*}$ is the solution to

$$
\frac{\phi_{S} h_{S} \sigma^{2}}{\left(\frac{h_{S}}{L} P_{S}^{*}+\sigma^{2}\right)^{2}}+\frac{\left(1-\phi_{S}\right) \sqrt{h_{S} L E_{M}}}{\sqrt{P_{S}^{*} \sqrt{\phi_{M} h_{M}}}}=E_{S}
$$

Proof: Define $u_{i, j}$ as the utility of selfish node $i=S$ or malicious node $i=M$ with a selfish opponent $j=S$ or malicious opponent $j=M$. The equilibrium strategies (23)-(25) follow from applying the KKT conditions (11) separately to the objective functions $\bar{u}_{S}=\phi_{S} u_{S, S}\left(P_{S}, P_{S}\right)+$ $\left(1-\phi_{S}\right) u_{S, M}\left(P_{S}, P_{M}\right)$ of each selfish node and $\bar{u}_{M}=$ $\phi_{M} u_{M, S}\left(P_{S}, P_{M}\right)+\left(1-\phi_{M}\right) u_{M, M}\left(P_{M}, P_{M}\right)$ of each malicious node, with constraint $P_{S} \geq 0$ and $P_{M} \geq 0$, where the utilities $u_{S, S}$ and $u_{S, M}$ are given by (3), and the utilities $u_{M, S}$ and $u_{M, M}$ are given by (4). 
Consider a selfish node with a malicious opponent. The equilibrium SINR of the selfish node is given by

$$
\gamma_{S}=\left\{\begin{array}{cc}
\frac{\sqrt{P_{S}} \sqrt{h_{S} L E_{M}}}{\sqrt{\phi_{M} h_{M}}}, & \text { if } P_{S}>\frac{L E_{M} \sigma^{4}}{\phi_{M} h_{S} h_{M}}, \\
\frac{h_{S} P_{S}}{\sigma^{2}}, & \text { otherwise, }
\end{array}\right.
$$

which decreases with $\phi_{S}$ for fixed $\phi_{M}$, with $\phi_{M}$ for fixed $\phi_{S}$ and with $\phi_{S}=\phi_{M}$. The expected SINR value $\gamma_{S}$ (averaged over the distribution of the opponent's type) decreases with $\phi_{S}=\phi_{M}$, as shown in Figure 3, under the assumption that the type belief distributions are both equal to the true distribution $\tilde{\phi}$ that any given node is selfish. In Figure 4, we evaluate the effect of the mismatch between the type belief distribution $\phi_{S}=\phi_{M}$ and true distribution $\tilde{\phi}$ on the equilibrium SINR $\gamma_{S}$ of any selfish node. The results show that assuming type beliefs other than the true distribution may possibly improve the equilibrium SINR value $\gamma_{S}$.

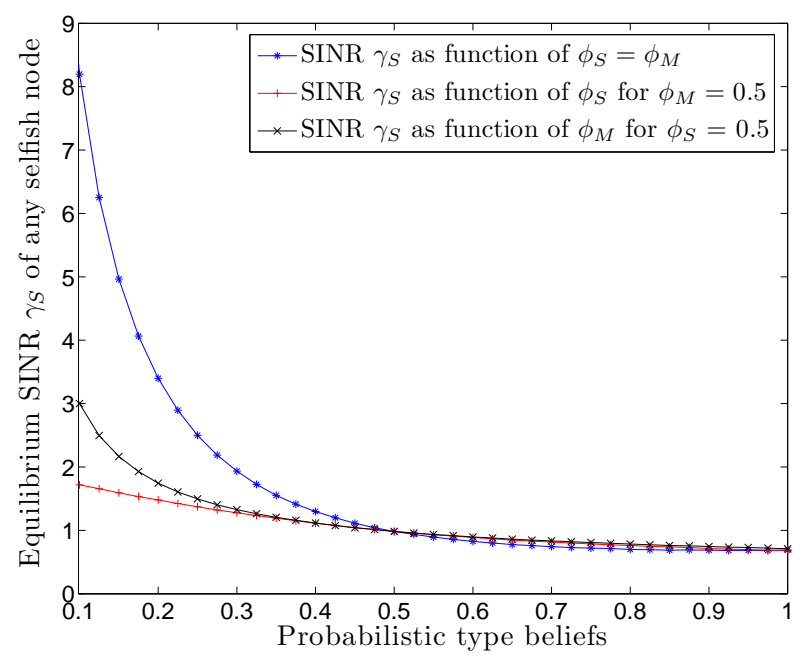

Fig. 3. The equilibrium SINR $\gamma_{S}$ of any selfish node as function of type belief probabilities for $h_{i}=1, E_{i}=1, i=1,2, L=1$ and $\sigma^{2}=0.1$.

Hence, the type uncertainty is beneficial for the selfish node and it should hide its type to increase $\gamma_{S}$, whereas the success of any malicious attack increases with $\phi_{S}$ and $\phi_{M}$.

\section{INTERACTIONS OF SELFISH AND MALiCIOUS TRANSMITTERS FOR REWARD FUNCTION}

$$
f_{i}\left(\gamma_{i}\right)=\log \left(1+\gamma_{i}\right)
$$

The analysis for reward function $f_{i}\left(\gamma_{i}\right)=\log \left(1+\gamma_{i}\right)$ is similar to the previous analysis for reward function $f_{i}\left(\gamma_{i}\right)=$ $\gamma_{i}$. Here, we outline the equilibrium strategies in Theorems 6-10. The proofs of Theorems 6-10 follow from the same arguments as in Theorems 1-5, where the reward function $f_{i}\left(\gamma_{i}\right)=\gamma_{i}$ is replaced by $f_{i}\left(\gamma_{i}\right)=\log \left(1+\gamma_{i}\right)$.

\section{A. Known Types of Two Transmitters}

Theorem 6: For two selfish transmitters, the unique Nash equilibrium strategies are

$$
P_{i}=\frac{\frac{h_{i} L}{E_{i}}-\frac{h_{j}}{E_{j}}-\sigma^{2}(L-1)}{h_{i}\left(L-\frac{1}{L}\right)}, j \neq i, i=1,2,
$$

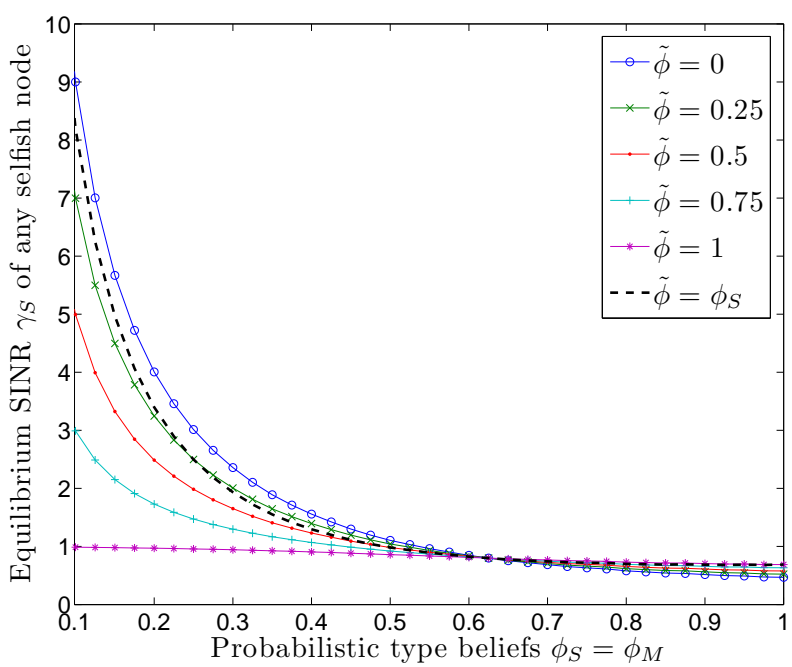

Fig. 4. The equilibrium SINR $\gamma_{S}$ of any selfish node as function of type belief probabilities for different values of true distribution $\tilde{\phi}$ that a node is selfish and for $h_{i}=1, E_{i}=1, i=1,2, L=1$ and $\sigma^{2}=0.1$.

if $\frac{h_{i} L}{E_{i}}-\frac{h_{j}}{E_{j}} \geq \sigma^{2}(L-1), i=1,2$,

$$
P_{i}=0, \quad P_{j}=\left[\frac{1}{E_{j}}-\frac{\sigma^{2}}{h_{j}}\right]^{+}, \quad j \neq i,
$$

if $\frac{h_{i} L}{E_{i}}-\frac{h_{j}}{E_{j}}<\sigma^{2}(L-1)$ and $\frac{h_{j} L}{E_{j}}-\frac{h_{i}}{E_{i}}>\sigma^{2}(L-1), j \neq i$.

The same utility function has been considered in [3] for selfish nodes. The equilibrium SINR of a selfish node $i$ is

$\gamma_{i}=\left\{\begin{array}{cc}\frac{\left(L^{2}-1\right) h_{i} P_{i}}{\frac{h_{j} L}{E_{j}}-\frac{h_{i}}{E_{i}}+\sigma^{2} L(L-1)}, & \text { if } \frac{h_{i} L}{E_{i}}-\frac{h_{j}}{E_{j}} \geq \sigma^{2}(L-1), \\ \frac{h_{i} P_{i}}{\sigma^{2}}, & \text { otherwise. }\end{array}\right.$

Theorem 7: For selfish transmitter 1 and malicious transmitter 2, the unique Nash equilibrium strategies are

$$
\begin{aligned}
P_{1} & =\frac{L E_{2} h_{1}}{E_{1}\left(E_{1} h_{2}+L E_{2} h_{1}\right)}, \\
P_{2} & =\frac{L}{h_{2}}\left(h_{1}\left(\frac{1}{E_{1}}-P_{1}\right)-\sigma^{2}\right),
\end{aligned}
$$

if $\frac{\sigma^{2} E_{1}}{h_{1}}+\frac{L E_{2} h_{1}}{E_{1} h_{2}+L E_{2} h_{1}} \leq 1$. Otherwise,

$$
P_{1}=\left[\frac{1}{E_{1}}-\frac{\sigma^{2}}{h_{1}}\right]^{+}, \quad P_{2}=0 .
$$

The equilibrium SINR of selfish node 1 is

$\gamma_{1}=\left\{\begin{array}{cc}\frac{L E_{2} h_{1}}{E_{1} h_{2}}, & \text { if } \frac{\sigma^{2} E_{1}}{h_{1}}+\frac{L E_{2} h_{1}}{E_{1} h_{2}+L E_{2} h_{1}} \leq 1, \\ {\left[\frac{h_{1}}{\sigma^{2} E_{1}}-1\right]^{+},} & \text {otherwise. }\end{array}\right.$

The malicious attack of node 2 is more successful in reducing $\gamma_{1}$ compared to the alternative selfish behavior, if the SINR value $\gamma_{1}$ given in (33) is smaller than the SINR value $\gamma_{i}$ given in (29) for $i=1$. This condition strongly depends on the underlying system parameters.

For two malicious nodes, the Nash equilibrium strategies are given by $P_{1}=0$ and $P_{2}=0$. 
B. Selfish Transmitter 1 (Known Type) and Transmitter 2 of Unknown Type

Theorem 8: For $\phi_{1} \in(0,1)$, the Bayesian Nash equilibrium strategies are

$$
P_{1}=\left[P_{1}^{*}\right]^{+}, \quad P_{2, S}=\left[P_{2, S}^{*}\right]^{+}, \quad P_{2, M}=\left[P_{2, M}^{*}\right]^{+},
$$

where $P_{1}^{*}, P_{2, S}^{*}$ and $P_{2, M}^{*}$ are solutions to

$$
\begin{array}{r}
\frac{h_{1} P_{1}^{*}}{L}+h_{2, S} P_{2, S}^{*}+\sigma^{2}=\frac{h_{2, S}}{E_{2, S}}, \\
\frac{h_{2, M} h_{1} P_{1}^{*}}{L}=E_{2, M}\left(\frac{h_{2, M} P_{2, M}^{*}}{L}+\sigma^{2}\right) \\
\times\left(\frac{h_{2, M} P_{2, M}^{*}}{L}+h_{1} P_{1}^{*}+\sigma^{2}\right), \\
\frac{\phi_{1} h_{1}\left(\frac{h_{2, S} P_{2, S}^{*}}{L}+\sigma^{2}\right)}{\frac{h_{2, S} P_{2, S}^{*}}{L}+h_{1} P_{1}^{*} \sigma^{2}} \\
+\frac{\left(1-\phi_{1}\right) h_{1}\left(\frac{h_{2, M} P_{2, M}^{*}}{L}+\sigma^{2}\right)}{\frac{h_{2, M} P_{2, M}^{*}}{L}+h_{1} P_{1}^{*}+\sigma^{2}}=E_{1} .
\end{array}
$$

In Nash equilibrium, the type uncertainty is better for selfish node 1 (i.e., selfish node hides its type), if $h_{1}$ is small and $E_{1}$ is large, or it is better for malicious node 2, otherwise.

\section{Malicious Transmitter 1 (Known Type) and Transmitter 2 of Unknown Type}

Theorem 9: For $\phi_{1} \in(0,1)$, the Bayesian Nash equilibrium strategies $P_{1} \geq 0, P_{2, S} \geq 0$ and $P_{2, M} \geq 0$ are

$$
\begin{aligned}
P_{1} & =\frac{L}{h_{1}}\left[\frac{h_{2, S}}{E_{2, S}}-h_{2, S} P_{2, S}-\sigma^{2}\right]^{+}, \\
P_{2, S} & =\frac{E_{1} h_{2, S} L}{E_{2, S}\left(L E_{1} h_{2, S}+\phi_{1} E_{2, S} h_{1}\right)}, \\
P_{2, M} & =0 .
\end{aligned}
$$

As $\phi_{1}$ decreases, $P_{1}$ decreases and $P_{2, S}$ increases from the equilibrium strategies (38)-(39). Consequently, the equilibrium SINR $\gamma_{2, S}$ of selfish node 2 increases, as $\phi_{1}$ decreases, i.e., the type uncertainty is beneficial for the selfish node of unknown type.

\section{Transmitters 1 and 2 of Unknown Types}

Theorem 10: For $\phi_{S} \in(0,1)$ and $\phi_{M} \in(0,1)$, the symmetric Bayesian Nash equilibrium strategies are

$$
P_{S}=\left[P_{S}^{*}\right]^{+}, \quad P_{M}=\left[P_{M}^{*}\right]^{+},
$$

where $P_{S}^{*}$ and $P_{M}^{*}$ are solutions to

$$
\begin{array}{r}
\frac{P_{S}^{*}}{\left(\frac{h_{M} P_{M}^{*}}{L}+\sigma^{2}\right)\left(\frac{h_{M} P_{M}^{*}}{L}+h_{S} P_{S}^{*}+\sigma^{2}\right)}=\frac{L E_{M}}{\phi_{M} h_{M} h_{S}}, \\
\frac{\phi_{S} h_{S} \sigma^{2}}{\left(\frac{h_{S} P_{S}^{*}}{L}+\sigma^{2}\right)\left(\frac{h_{S} P_{S}^{*}}{L}+h_{S} P_{S}^{*} \sigma^{2}\right)} \\
\quad+\frac{\left(1-\phi_{S}\right) h_{S}}{\frac{h_{M} P_{M}^{*}}{L}+h_{S} P_{S}^{*}+\sigma^{2}}=E_{S} .
\end{array}
$$

For $h_{S}=h_{M}=1, E_{S}=E_{M}=1, L=1$ and $\sigma^{2}=0$, the equilibrium transmission powers from (41)-(43) are given by $P_{S}=\frac{\left(1-\phi_{S}\right)^{2}}{1-\phi_{S}+\phi_{M}}$ and $P_{M}=\frac{\left(1-\phi_{S}\right) \phi_{M}}{1-\phi_{S}+\phi_{M}}$ for selfish and malicious nodes, respectively. If the opponent is malicious, the equilibrium SINR value of selfish node is given by $\gamma_{S}=$ $\frac{1-\phi_{S}}{\phi_{M}}$, which decreases with both $\phi_{S}$ and $\phi_{M}$, i.e., any selfish node prefers type uncertainty and should hide its type to increase the SINR value $\gamma_{S}$.

\section{BAYESIAN LEARNING OF SELFISH AND MALICIOUS User Types in Power-Controlled MAC}

Next, we deviate from the previous focus of fixed and known type belief distributions and extend the analysis to the dynamic situation in which nodes learn each other's types based on the outcomes of the power-controlled MAC game at each time slot. Consider the reward function $f\left(\gamma_{S}\right)=\gamma_{S}$ for unknown types of two transmitters.

For simplicity, assume $h_{S}=h_{M}=1, E_{S}=E_{M}=1$, $L=1$ and $\sigma^{2}=0$. Selfish and malicious nodes play the Nash equilibrium strategies $P_{S}\left(\phi_{S}, \phi_{M}\right)=\frac{\left(1-\phi_{S}\right)^{2}}{\phi_{M}}$ and $P_{M}\left(\phi_{S}, \phi_{M}\right)=1-\phi_{S}$ from (23)-(25). Selfish and malicious nodes update $\phi_{S}$ and $\phi_{M}$, respectively, based on the opponent's power (which can be observed through the SINR feedback sent from the receiver back to the transmitters).

Nodes assume that the belief of the opponent with the opposite type is uniformly distributed over $[0,1]$, whereas nodes of the same type have the same belief distribution and update their beliefs according to the same rule. Define $P_{o}^{(k)}$ as the opponent's power and $\phi_{S}^{(k)}$ as the value of $\phi_{S}$ at the $k$ th iteration. Selfish and malicious nodes update their beliefs $\phi_{S}$ and $\phi_{M}$, respectively, on the opponent's type according to the Bayes' rule:

$$
\begin{aligned}
\phi_{S}^{(k+1)} & =\frac{\phi_{S}^{(k)} a_{S}^{(k)}\left(P_{o}^{(k)}\right)}{\phi_{S}^{(k)} a_{S}^{(k)}\left(P_{o}^{(k)}\right)+\left(1-\phi_{S}^{(k)}\right) b_{S}^{(k)}\left(P_{o}^{(k)}\right)}, \\
\phi_{M}^{(k+1)} & =\frac{\phi_{M}^{(k)} a_{M}^{(k)}\left(P_{o}^{(k)}\right)}{\phi_{M}^{(k)} a_{M}^{(k)}\left(P_{o}^{(k)}\right)+\left(1-\phi_{M}^{(k)}\right) b_{M}^{(k)}\left(P_{o}^{(k)}\right)},
\end{aligned}
$$

where $a_{i}^{(k)}\left(P_{o}^{(k)}\right)=P\left(P_{o}^{(k)} \mid\right.$ the opponent of node $i$ is selfish $)$ and $b_{i}^{(k)}\left(P_{o}^{(k)}\right)=P\left(P_{o}^{(k)} \mid\right.$ the opponent of node $i$ is malicious $)$ for $i \in\{S, M\}$ can be computed as

$$
\begin{array}{r}
a_{S}^{(k)}\left(P_{o}^{(k)}\right)=\left\{\begin{array}{l}
0, P_{o}^{(k)}<\left(1-\phi_{S}^{(k)}\right)^{2}, \\
1, P_{o}^{(k)} \geq\left(1-\phi_{S}^{(k)}\right)^{2},
\end{array}\right. \\
b_{S}^{(k)}\left(P_{o}^{(k)}\right)=b_{M}^{(k)}\left(P_{o}^{(k)}\right)=\left\{\begin{array}{l}
0, P_{o}^{(k)}>1, \\
1, P_{o}^{(k)} \leq 1,
\end{array}\right. \\
a_{M}^{(k)}\left(P_{o}^{(k)}\right)=\left\{\begin{array}{c}
\frac{1}{3\left(P_{o}^{(k)}\right)^{2}}, P_{o}^{(k)}>1, \\
\frac{1}{3 \sqrt{P_{o}^{(k)}},} P_{o}^{(k)} \leq 1 .
\end{array}\right.
\end{array}
$$

A selfish node eventually detects the type of the selfish opponent with power $P_{o}^{(k)}$, whenever $P_{o}^{(k)}>1$ is observed, or it detects the type of the malicious opponent, whenever $P_{o}^{(k)}<\left(1-\phi_{S}^{(k)}\right)^{2}$ is observed. From (44)-(45), we have $\phi_{S}^{(k+1)}=\phi_{S}^{(0)}$, until the type of the opponent is detected. The 
number of iterations to detect the opponent as a selfish or malicious node is a geometric random variable with success probability $\left(1-\phi_{S}^{(0)}\right)^{2}$.

On the other hand, a malicious node eventually detects the type of selfish opponent, whenever $P_{o}^{(k)}>1$ is observed or whenever the selfish node detects the type of malicious opponent at the previous iteration (and updated its transmission power accordingly). Then, the expected number of iterations to detect the opponent as a selfish node can be computed as $p+\frac{1}{1-p}\left[\frac{1}{1-(1-p)^{2}}-\left(1-(1-p)^{2}\right)\right]$, where $p=\left(1-\phi_{S}^{(0)}\right)^{2}$, and the average detection time grows with $\phi_{S}^{(0)}$.

However, a malicious node cannot detect the type of a malicious opponent with probability one at a particular iteration, but the probability $\phi_{M}^{(k)}$ asymptotically approaches 0 according to the Bayesian update mechanism (45), as the number of time iterations $k$ increases. Figure 5 shows the updates of the malicious node's type belief probability $\phi_{M}^{(k)}$ averaged over the distribution of the opponent's power $P_{O}^{(k)}$. Note that the probability $\phi_{M}^{(k)}$ converges with smaller rate, if the malicious node selects a larger value for the initial type belief probability $\phi_{M}^{(0)}$ that the opponent is selfish.

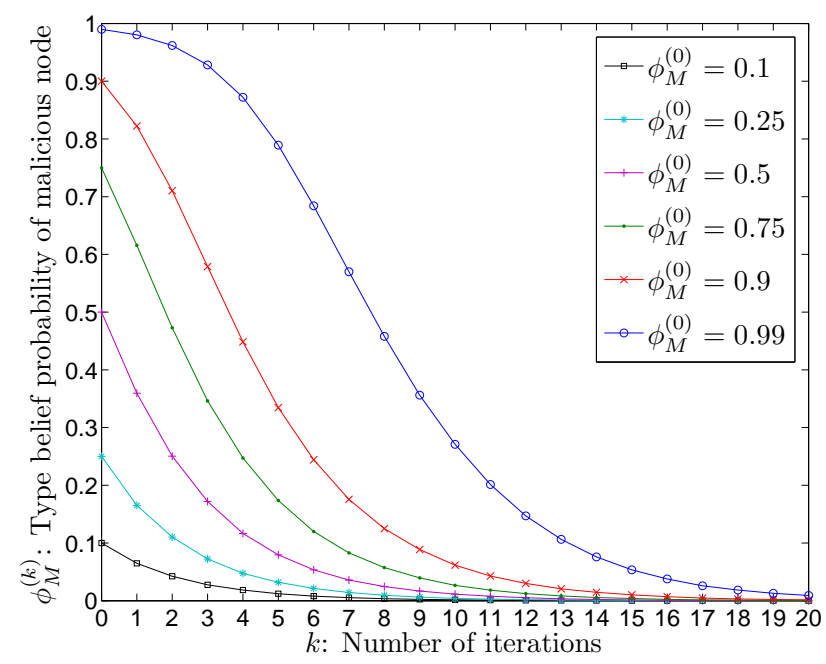

Fig. 5. The type belief probability $\phi_{M}^{(k)}$ of a malicious node with a malicious opponent for $f\left(\gamma_{S}\right)=\gamma_{S}, h_{S}=h_{M}=1, E_{S}=E_{M}=1$, $L=1$ and $\sigma^{2}=0$.

\section{Power-Controlled MAC Game For Arbitrary NuMBER OF SELFISH AND MALICIOUS TRANSMITTERS}

Let $n$ and $n_{s}$ denote the total number of nodes and the number of selfish nodes, respectively, where $n \leq n_{\max }$ and $n_{\max }$ is the maximum possible number of nodes in the system. Let $\eta_{S}\left(n, n_{s}\right)$ and $\eta_{M}\left(n, n_{s}\right)$ denote the joint probability mass function of $n$ and $n_{s}$, as believed by a selfish and malicious node, respectively. Consider common parameters (known by all nodes), as defined in Section III-D, and assume symmetric strategies $P_{S}$ or $P_{M}$ depending on whether the node is selfish or malicious. For the common reward function $f_{i}(\gamma)=f(\gamma)$ of any selfish node $i$, the utilities of selfish and malicious nodes are given by

$$
\begin{aligned}
u_{S}\left(P_{S}, P_{M}\right)= & \sum_{n=1}^{n_{\max }} \sum_{n_{s}=1}^{n} \eta_{S}\left(n, n_{s}\right) f\left(\frac{h_{S} P_{S}}{\psi\left(n_{s}\right)}\right) \\
& -P_{S} E_{S}, \\
u_{M}\left(P_{S}, P_{M}\right)= & -\sum_{n=1}^{n_{\max }} \sum_{n_{s}=0}^{n-1} \eta_{M}\left(n, n_{s}\right) n_{s} f\left(\frac{h_{S} P_{S}}{\psi\left(n_{s}\right)}\right) \\
& -P_{M} E_{M},
\end{aligned}
$$

respectively, where $\psi(\tilde{n})=\frac{1}{L}\left((\tilde{n}-1) h_{S} P_{S}+(n-\right.$ $\left.\tilde{n}) h_{M} P_{M}\right)+\sigma^{2}$. Any malicious node minimizes the sum of throughput rewards of all $n_{s}$ possibly selfish nodes subject to the additional objective of minimizing the energy cost.

Theorem 11: The symmetric Bayesian Nash equilibrium strategies $P_{S}$ and $P_{M}$ of selfish and malicious transmitters are

$$
P_{S}=\left[P_{S}^{*}\right]^{+}, \quad P_{M}=\left[P_{M}^{*}\right]^{+},
$$

where $P_{M}^{*}$ and $P_{S}^{*}$ are solutions to

$$
\begin{gathered}
\frac{E_{S}}{h_{S}}=\sum_{n=1}^{n_{\max }} \sum_{n_{s}=1}^{n} \eta_{S}\left(n, n_{s}\right)\left(\frac{\frac{1}{L}\left(n-n_{s}\right) h_{M} P_{M}^{*}+\sigma^{2}}{\psi\left(n_{s}\right)\left(\psi\left(n_{s}\right)+\zeta\right)}\right), \\
\frac{E_{M} L}{h_{S} h_{M}}=\sum_{n=1}^{n_{\max }} \sum_{n_{s}=0}^{n-1} \eta_{M}\left(n, n_{s}\right)\left(\frac{n_{s}\left(n-n_{s}\right) P_{S}^{*}}{\psi\left(n_{s}\right)\left(\psi\left(n_{s}\right)+\zeta\right)}\right)
\end{gathered}
$$

for perfect information of type beliefs, where $\psi(\tilde{n})=$ $\frac{1}{L}\left((\tilde{n}-1) h_{S} P_{S}^{*}+(n-\tilde{n}) h_{M} P_{M}^{*}\right)+\sigma^{2}, \zeta=0$ for $f\left(\gamma_{S}\right)=\gamma_{S}$ and $\zeta=h_{S} P_{S}$ for $f\left(\gamma_{S}\right)=\log \left(1+\gamma_{S}\right)$.

Proof: The equilibrium strategies (51)-(53) follow from applying the KKT conditions (generalized to arbitrary number of nodes) separately to the objective functions $u_{S}\left(P_{S}, P_{M}\right)$ of selfish nodes and $u_{M}\left(P_{S}, P_{M}\right)$ of malicious nodes, with constraints $P_{S} \geq 0$ and $P_{M} \geq 0$, where the utilities $u_{S}$ and $u_{M}$ are given by (49)-(50), respectively.

The equilibrium SINR of any selfish node is given by $\gamma_{S}=\frac{h_{S} P_{S}}{\psi\left(n_{s}\right)}$. Assume $E_{S} h_{M}=E_{M} h_{S}$ and $L=1$. For both reward functions $f\left(\gamma_{S}\right)=\gamma_{S}$ and $f\left(\gamma_{S}\right)=\log \left(1+\gamma_{S}\right)$, the equilibrium value of $\gamma_{S}$ for given $n$ decreases first with the small values of $n_{s}$, then reaches the minimum for the intermediate values $n_{s}$, and finally increases with the large values of $n_{s}$. There exists a critical value for the number of malicious nodes beyond which $\gamma_{S}$ increases again, i.e., a malicious attack is more successful, if it is accompanied with the selfish behavior of other nodes. The total throughput reward $n_{s} \gamma_{S}$ increases monotonically with the number of selfish nodes $n_{s}$, as shown in Figure 6.

\section{RANDOM ACCESS GAMES FOR SELFISH AND \\ MALicious TRANSMitTERS OF UNKNOWN TYPES}

The results for power control game with incomplete type information generalize to other MAC models. Next, consider random access games, where nodes choose between transmitting and waiting. Assume a synchronous slotted system with collision channels such that more than one simultaneous transmission fails. The primary focus is again on the case 


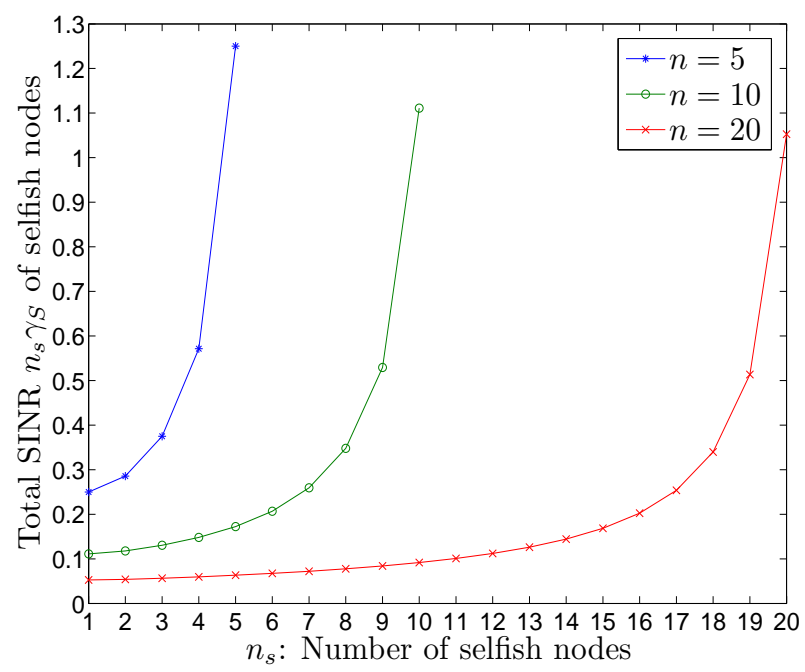

Fig. 6. The total SINR $n_{s} \gamma_{S}$ of selfish nodes in Nash equilibrium as function of $n_{s}$ for $E_{S} h_{M}=E_{M} h_{S}$ and $L=1$.

of two transmitters. Define $p_{i} \in[0,1]$ as transmission probability and $E_{i} \in(0,1)$ as energy cost (per transmission) of node $i=1,2$. Any selfish node $i$ receives unit throughput reward for successful transmission. The expected utility of selfish node $i$ is given by

$$
u_{i}\left(p_{1}, p_{2}\right)=p_{i}\left[-E_{i}+1-p_{j}\right]+\left(1-p_{i}\right)[0], j \neq i,
$$

for $i=1,2$. Any malicious node $i$ incurs a unit cost, if the opponent is selfish and successfully transmits at the given time slot. The expected utility of malicious node $i$ is given by

$$
u_{i}\left(p_{1}, p_{2}\right)=\left\{\begin{array}{c}
p_{i}\left[-E_{i}\right]+\left(1-p_{i}\right)\left[-p_{j}\right] \\
\text { if the opponent node } j \text { is selfish } \\
p_{i}\left[-E_{i}\right]+\left(1-p_{i}\right)[0] \\
\text { if the opponent node } j \text { is malicious }
\end{array}\right.
$$

for $j \neq i, i=1,2$.

Theorem 12: The symmetric Bayesian Nash equilibrium strategies $p_{S}$ and $p_{M}$ of selfish and malicious transmitters are

$$
\begin{array}{r}
p_{S}=1, p_{M}=0, \text { if } \phi_{S}<1-E_{S}, \phi_{M}<E_{M}, \\
p_{S}=\frac{1-E_{S}}{\phi_{S}}, p_{M}=0, \\
\text { if } \phi_{S}>1-E_{S}, E_{M} \phi_{S}>\phi_{M}\left(1-E_{S}\right), \\
p_{S}=\frac{E_{M}}{\phi_{M}}, p_{M}=\frac{1-E_{S}-E_{M} \frac{\phi_{S}}{\phi_{M}}}{1-\phi_{S}}, \\
\text { if } \phi_{S}<E_{S}+E_{M} \frac{\phi_{S}}{\phi_{M}}<1, \phi_{M}>E_{M} .
\end{array}
$$

Proof: (a) The expected utility of a given selfish node $u_{S}\left(p_{S}, p_{M}\right)=p_{S}\left(-E_{S}+\phi_{S}\left(1-p_{S}\right)+\left(1-\phi_{S}\right)\left(1-p_{M}\right)\right)$ is maximized by transmitting, i.e., $p_{S}=1$, if $\left(1-\phi_{S}\right)(1-$ $\left.p_{M}\right)>E_{S}$. Given $p_{S}=1$, the expected utility of a malicious node $u_{M}\left(p_{S}, p_{M}\right)=p_{M}\left(-E_{M}\right)+\left(1-p_{M}\right)\left(-\phi_{M} p_{S}\right)$ is maximized by waiting, i.e., $p_{M}=0$, if $\phi_{M}<E_{M}$, or by transmitting, if $\phi_{M}>E_{M}$. However, $u_{S}\left(p_{S}, 1\right)$ is not maximized by $p_{S}=1$ (therefore the strategy pair $p_{S}=1$ and $p_{M}=1$ does not yield a Nash equilibrium), whereas $u_{S}\left(p_{S}, 0\right)$ is maximized by $p_{S}=1$, if $1-\phi_{S}>E_{S}$ such that the Nash equilibrium strategy (56) follows.

The utility of a selfish node $u_{S}\left(p_{S}, p_{M}\right)$ is maximized by waiting, i.e., $p_{S}=0$, if $\left(1-\phi_{S}\right)\left(1-p_{M}\right)<E_{S}$. Given $p_{S}=$ 0 , the utility of a malicious node $u_{M}\left(p_{S}, p_{M}\right)$ is maximized by waiting only, i.e., $p_{M}=0$. However, $u_{S}\left(p_{S}, 0\right)$ cannot be maximized by $p_{S}=0$, and therefore the strategy $p_{S}=0$ does not yield a Nash equilibrium.

(b) Given $p_{M}=0$, the utility of a selfish node $u_{S}\left(p_{S}, p_{M}\right)$ is maximized by transmitting, i.e., $p_{S}=1$, if $1-E_{S}-$ $\phi_{S}>0$. This corresponds to Nash equilibrium strategy (56). The strategy $p_{S}=1$ cannot yield Nash equilibrium, since it violates the condition $E_{S}<1$. The utility $u_{S}\left(p_{S}, p_{M}\right)$ is indifferent to $p_{S}$, if $p_{S}=\frac{1-E_{S}}{\phi_{S}}$. Given $p_{S}=\frac{1-E_{S}}{\phi_{S}}$, the utility of a malicious node $u_{M}\left(p_{S}, p_{M}\right)$ is maximized by waiting, i.e., $p_{M}=0$, if $E_{M} \phi_{S}>\phi_{M}\left(1-E_{S}\right)$ such that the Nash equilibrium strategy (57) follows. For $p_{S}=\frac{1-E_{S}}{\phi_{S}}$, $p_{M}=1$ cannot yield any Nash equilibrium, since $u_{S}\left(p_{S}, 1\right)$ is maximized only by $p_{S}=0$ provided that $E_{S}>\phi_{S}$ and $u_{M}\left(0, p_{M}\right)$ cannot be maximized by $p_{M}=1$.

(c) Consider mixed strategies such that selfish and malicious nodes are indifferent to $p_{S}$ and $p_{M}$, respectively, to maximize $u_{S}$ and $u_{M}$. From $u_{S}\left(1, p_{M}\right)=u_{S}\left(0, p_{M}\right)$ and $u_{M}\left(p_{S}, 1\right)=u_{M}\left(p_{S}, 0\right)$, we obtain the equilibrium strategy (58) subject to $0 \leq p_{S} \leq 1$ and $0 \leq p_{M} \leq 1$.

If the type belief distributions $\phi_{S}=\phi_{M}$ are equal to the true probability that a node is selfish, the resulting throughput rates are $\lambda_{S}=1-\phi_{S}, \lambda_{S}=\frac{E_{S}\left(1-E_{S}\right)}{\phi_{S}}$ and $\lambda_{S}=E_{M}(1+$ $\left.\frac{E_{S}}{\phi_{S}}\right)$ for the strategies (56)-(58), respectively.

Consider a selfish node with malicious opponent. The throughput rates are $\lambda_{S}=1, \lambda_{S}=\frac{1-E_{S}}{\phi_{S}}$ and $\lambda_{S}=$ $\frac{E_{M}}{\phi_{M}}\left(\frac{E_{S}+E_{M} \frac{\phi_{S}}{\phi_{M}}-\phi_{S}}{1-\phi_{S}}\right)$ for the strategies (56)-(58), respectively. If the types are known, the equilibrium strategies are $p_{S}=E_{M}$ and $p_{M}=1-E_{S}$ with throughput $\lambda_{S}=E_{M} E_{S}$. Then, the strategy (56) achieves higher throughput. It is beneficial for the selfish node to hide its type such that the strategy (57) is more throughput-efficient, if $E_{S}\left(1+E_{M} \phi_{S}\right)<1$, which holds for small energy costs, and the strategy (58) is more throughput-efficient, if $E_{S}\left(1-\phi_{M}\left(1-\phi_{S}\right)\right)>$ $\phi_{S}\left(1-\frac{E_{M}}{\phi_{M}}\right)$, which holds for large energy costs.

Selfish and malicious nodes can further choose $\phi_{S}$ and $\phi_{M}$, respectively, to maximize and minimize the throughput $\lambda_{S}$ of a selfish node. The resulting equilibrium probabilities are $\phi_{M}^{*}=1$ and $\phi_{S}^{*}=0$, if $E_{S}+E_{M}<1$, with throughput $\lambda_{S}=E_{S} E_{M}$, or $\phi_{S}^{*}=\frac{1-E_{S}}{E_{M}}$, if $E_{S}+E_{M}>1$, with throughput $\lambda_{S}=E_{M}$. Hence, the equilibrium throughput $\lambda_{S}$ can be improved, if $E_{S}+E_{M}<1$, compared to the case of known types with equilibrium throughput $\lambda_{S}=E_{S} E_{M} \cdot{ }^{3}$

\footnotetext{
${ }^{3}$ The receiver can identify the type of any node (i.e., whether the received packet carries real data or constitutes to a jamming signal), if it is the only one transmitting at the given time slot. Then, we can consider a Markov game formulation such that the strategy of a node depends on its type and the state of the game that is updated, whenever the type of any node is detected by the receiver.
} 


\section{A. Unknown Type Belief Distributions and Energy Costs}

Assume that node $i$ does not know the exact values of type belief probability $\phi_{j}$ and energy cost $E_{j}$ of the opponent node $j$, but knows their distributions. The uncertainties on node types and energy costs are jointly formulated as probabilistic beliefs. Define $\mu_{i}$ as the subjective belief (namely the probability distribution) of node $i=1,2$ about the parameters $\phi_{j}$ and $E_{j}$ of the opponent $j \neq i$. Consider the symmetric game model such that any selfish node has belief $\mu_{S}$ and any malicious node has belief $\mu_{M}$.

Theorem 13: The Nash equilibrium strategy of a selfish or malicious node is to transmit, respectively, if and only if

$$
\begin{array}{r}
\theta_{S}=\frac{E_{S}}{1-\phi_{S}}<\theta_{S}^{*}=1-\mu_{S}\left(\theta_{M}<\theta_{M}^{*}\right), \text { or } \\
\theta_{M}=\frac{E_{M}}{\phi_{M}}<\theta_{M}^{*}=\mu_{M}\left(\theta_{S}<\theta_{S}^{*}\right) .
\end{array}
$$

Proof: Each node assumes that the opponent node of the same type would make the same decision to transmit or to wait. Any selfish node transmits, if $u_{S}\left(1, p_{M}\right)>u_{S}\left(0, p_{M}\right)$, where $u_{S}$ is given by (54), i.e., if $E_{S}<\left(1-\phi_{S}\right)\left(1-p_{M}\right)$, or waits, if $E_{S}>\left(1-\phi_{S}\right)\left(1-p_{M}\right)$. In the case of equality, the selfish node either transmits or waits. This leads to monotonic transmission decision of selfish node such that $\theta_{S}=\frac{E_{S}}{1-\phi_{S}}<\theta_{S}^{*}$, where $\theta_{S}^{*}=1-p_{M}$. Selfish node does not know the transmission probability $p_{M}$ of malicious node but has subjective belief distribution for $p_{M}$, if the transmission decision of any malicious node also satisfies the monotonicity property in terms of type belief and cost parameters.

Any malicious node transmits, if $u_{M}\left(p_{S}, 1\right)>u_{M}\left(p_{S}, 0\right)$, where $u_{M}$ is given by (55), i.e., if $E_{M}<\phi_{M} p_{S}$, or waits, if $E_{M}>\phi_{M} p_{S}$. In the case of equality, the malicious node either transmits or waits. This also leads to monotonic transmission decisions of malicious node such that $\theta_{M}=$ $\frac{E_{M}}{\phi_{M}}<\theta_{M}^{*}$, where $\theta_{M}^{*}=p_{S}$. Malicious node does not know the transmission probability $p_{S}$ of selfish node but has subjective belief distribution for $p_{S}$, since the transmission decisions of selfish node are also monotonic in terms of type belief and cost parameters. The threshold parameters are $\theta_{S}^{*}=1-p_{M}^{*}$ with $p_{M}^{*}=\mu_{S}\left(\theta_{M}<\theta_{M}^{*}\right)$ and $\theta_{M}^{*}=p_{S}^{*}$ with $p_{S}^{*}=\mu_{M}\left(\theta_{S}<\theta_{S}^{*}\right)$ such that the monotonic transmission decisions (59)-(60) follow in Nash equilibrium.

If the opponent's beliefs on type probabilities $\phi_{S}$ and $\phi_{M}$ and costs $E_{S}$ and $E_{M}$ are independent and uniformly distributed over $[0,1]$ for each selfish and malicious node, the parameters $\theta_{S}=\frac{E_{S}}{1-\phi_{S}}$ and $\theta_{M}=\frac{E_{M}}{\phi_{M}}$ follow a uniform ratio distribution

$$
P\left(\theta_{i}=\theta\right)=\left\{\begin{array}{cc}
\frac{1}{2}, & 0<\theta<1, \\
\frac{1}{2 \theta^{2}}, & \theta \geq 1, \\
0, & \text { otherwise, }
\end{array} \quad i \in\{S, M\} .\right.
$$

From (59)-(60), there exist unique equilibrium thresholds $\theta_{S}^{*}=\frac{4}{5}$ and $\theta_{M}^{*}=\frac{2}{5}$. A selfish node with malicious opponent achieves equilibrium throughput $\lambda_{S}=\frac{8}{25}$, which is greater than the throughput $\lambda_{S}=\frac{1}{4}$ averaged over cost distributions for known types. Hence, the type uncertainty is beneficial for selfish nodes to increase the equilibrium throughput $\lambda_{S}$.

\section{B. Arbitrary Number of Selfish and Malicious Users}

Consider the probabilistic belief distributions $\eta_{S}\left(n, n_{s}\right)$ and $\eta_{M}\left(n, n_{s}\right)$ of selfish and malicious nodes to represent the total number of nodes $n$ and the number of selfish nodes $n_{s}$ (as defined in Section VI for power control). Assume a symmetric game model with transmission probability $p_{S}$ and $p_{M}$ for any selfish and malicious node, respectively. Any malicious node wishes to minimize the sum of selfish throughput rewards and incurs a unit cost for each successfully transmitting selfish node. The expected utility of a selfish node is

$$
\begin{array}{r}
u_{S}\left(p_{S}, p_{M}\right)=\sum_{n=1}^{n_{\max }} \sum_{n_{s}=1}^{n} \eta_{S}\left(n, n_{s}\right)\left(p _ { S } \left[-E_{S}\right.\right. \\
\left.\left.+\left(1-p_{S}\right)^{n_{s}-1}\left(1-p_{M}\right)^{n-n_{s}}\right]+\left(1-p_{S}\right)[0]\right)
\end{array}
$$

and the expected utility of a malicious node is

$$
\begin{array}{r}
u_{M}\left(p_{S}, p_{M}\right)=\sum_{n=1}^{n_{\max }} \sum_{n_{s}=0}^{n-1} \eta_{M}\left(n, n_{s}\right)\left(p_{M}\left[-E_{M}\right]\right. \\
\left.+\left(1-p_{M}\right)\left[-n_{s} p_{S}\left(1-p_{S}\right)^{n_{s}-1}\left(1-p_{M}\right)^{n-n_{s}-1}\right]\right) .
\end{array}
$$

Theorem 14: For perfect information of $\eta_{S}\left(n, n_{s}\right)$ and $\eta_{M}\left(n, n_{s}\right)$, the symmetric Bayesian Nash equilibrium strategies $p_{S}$ and $p_{M}$ of selfish and malicious transmitters are

$$
p_{S}=\min \left(\left[p_{S}^{*}\right]^{+}, 1\right), \quad p_{M}=\min \left(\left[p_{M}^{*}\right]^{+}, 1\right),
$$

where $p_{S}^{*}$ and $p_{S}^{*}$ are solutions to

$$
\begin{array}{r}
E_{S}=\sum_{n=1}^{n_{\max }} \sum_{n_{s}=1}^{n} \eta_{S}\left(n, n_{s}\right) \\
\times\left[\left(1-n_{S} p_{S}\right)\left(1-p_{S}\right)^{n_{s}-2}\left(1-p_{M}\right)^{n-n_{s}}\right], \\
E_{M}=\sum_{n=1}^{n_{\max }} \sum_{n_{s}=0}^{n-1} \eta_{M}\left(n, n_{s}\right) n_{s}\left(n-n_{s}\right) \\
\times p_{S}\left(1-p_{S}\right)^{n_{s}-1}\left(1-p_{M}\right)^{n-n_{s}-1} .
\end{array}
$$

Proof: The equilibrium strategies $p_{S}$ and $p_{M}$ from (64)-(66) follow from applying the KKT conditions separately to the objective functions $u_{S}\left(p_{S}, p_{M}\right)$ of any selfish node and $u_{M}\left(p_{S}, p_{M}\right)$ of any malicious node with constraints $0 \leq p_{S} \leq 1$ and $0 \leq p_{M} \leq 1$, where $u_{S}\left(p_{S}, p_{M}\right)$ and $u_{M}\left(p_{S}, p_{M}\right)$ are given by (62)-(63), respectively.

The throughput of any selfish node in Nash equilibrium is given by $\lambda_{S}=p_{S}\left(1-p_{S}\right)^{n_{s}-1}\left(1-p_{M}\right)^{n-n_{s}}$. Figure 7 evaluates $\lambda_{S}$ as function of $n$, where $n_{s}=1$, and shows that $\lambda_{S}$ decreases as the number of malicious nodes increases (except for small values of energy costs $E_{S}$ and $E_{M}$ and total number of nodes $n$ ).

Assume partial information on the probabilistic belief of the opponent on the values of $n$ and $n_{s}$. Let $n(i)$ and $n_{s}(i)$ denote the total number of nodes and the number of selfish nodes believed by any selfish node $i=S$ or by any malicious node $i=M$. Let $\mathcal{P}_{i}\left(n, n_{s}\right)$ denote the probability distribution function that selfish or malicious node $i \in\{S, M\}$ believes to represent the belief of node $j \in\{S, M\}, j \neq i$, on the values of $n$ and $n_{s}$. 


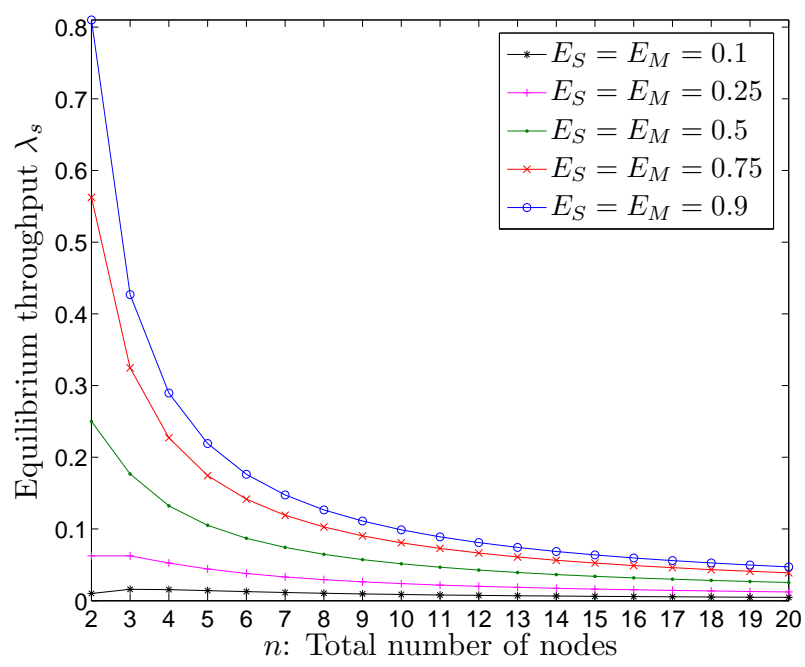

Fig. 7. The throughput $\lambda_{S}$ achievable by one selfish node in Nash equilibrium as function of $n$ for $n_{s}=1$.

Each node assumes that any other node of the same type would make the same transmission decision. Any selfish node transmits (i.e., $\left.p_{S}=1\right)$, if $u_{S}\left(1, p_{M}\right)>u_{S}\left(0, p_{M}\right)$ in Eq. (62), i.e., if $\left(1-p_{M}\right)^{n-1}>E_{S}$ and $n_{s}=1$. On the other hand, any malicious node transmits (i.e., $p_{S}=1$ ), if $u_{M}\left(p_{S}, 1\right) \geq u_{M}\left(p_{S}, 0\right)$ in Eq. (63), i.e., if $n_{s} p_{S}(1-$ $\left.p_{S}\right)^{n_{s}-1}>E_{M}$. For incomplete information on node types, the transmission decisions in Nash equilibrium satisfy the following monotonicity properties:

Any selfish node transmits, if and only if

$$
n(S)<n^{*}(S), \quad n_{s}(S)=1,
$$

and any malicious node transmits, if and only if

$$
n_{s, 1}^{*}(M)<n_{s}(M)<n_{s, 2}^{*}(M),
$$

provided that the non-negative cut-off thresholds $n^{*}(S)$ and $n_{s, 2}^{*}(M)$ are the largest solutions and the non-negative cutoff threshold $n_{s, 1}^{*}(M)$ is the smallest solution to

$$
\begin{aligned}
\left(1-\varphi_{M}\right)^{n^{*}(S)-1} & \geq E_{S}, \\
n_{s, i}^{*}(M) \varphi_{S}\left(1-\varphi_{S}\right)^{n_{s, i}^{*}(M)} & \geq E_{M}, \quad i=1,2,
\end{aligned}
$$

where

$$
\begin{aligned}
& \varphi_{S}=\sum_{1 \leq n<n^{*}(S)} \mathcal{P}_{M}(n, 1), \\
& \varphi_{M}=\sum_{n_{s, 2}^{*}(M) \leq n} \sum_{n_{s, 1}^{*}(M)<n_{s}<n_{s, 2}^{*}(M)} \mathcal{P}_{S}\left(n, n_{s}\right) \text {. } \\
& \text { VIII. CONCLUSIONS }
\end{aligned}
$$

We presented game-theoretic models to establish a security paradigm at the MAC layer of wireless networks. For selfish and malicious users of unknown types, we considered different MAC models based on power control and random access, and derived the Nash equilibrium strategies depending on the degree of type uncertainty. The performance is measured in terms of throughput rewards, transmission energy costs and malicious attack incentives. The analysis showed under what conditions the type identities should be concealed or revealed to improve the individual performance as a selfish user or to reduce the system performance as a malicious user.

We also extended the results to incorporate different degrees of uncertainty in type distributions and cost parameters, and presented Bayesian learning mechanisms for the type belief updates. The analysis provides new insights into using the type uncertainty as an inherent defense mechanism against the denial of service attacks in wireless networks.

Future work should generalize the game model to multihop wireless networks. This would extend the denial of service attack possibilities to the network layer operations and require cross-layer design with the attack and defense mechanisms that have been established for the MAC layer.

\section{REFERENCES}

[1] H. Ji and C. Huang, "Non-cooperative Uplink Power Control in Cellular Radio Systems," Wireless Networks, vol. 4, no. 3, pp. 233240, Mar. 1998

[2] C. Saraydar, N. B. Mandayam, and D. J. Goodman, "Efficient Power Control via Pricing in Wireless Data Networks," IEEE Trans. Commun., vol. 50, no. 2, pp. 291-303, Feb. 2002.

[3] T. Alpcan, T. Basar, R. Srikant, and E. Altman, "CDMA Uplink Power Control as a Noncooperative Game," Wireless Networks, vol. 8, no. 6, pp. 659-670, Nov. 2002.

[4] A. B. MacKenzie and S. B. Wicker, "Game Theory and the Design of Self-Configuring, Adaptive Wireless Networks," IEEE Commun. Mag., vol. 39, no. 11 , pp. 126-131, Nov. 2001

[5] Y. Jin and G. Kesidis, "Equilibria of a Non-cooperative Game for Heterogeneous Users of an Aloha Network," IEEE Communications Letters, vol. 6, no. 7, pp. 282-284, July 2002.

[6] J.-W Lee, M. Chiang, and R. A. Calderbank, "Utility-Optimal Random-Access Control," IEEE Trans. Wireless Commun., vol. 6, no. 7, pp. 2741-2751, July 2007.

[7] J. Zander, "Jamming in Slotted ALOHA Multihop Packet Radio Networks," IEEE Trans. Commun., vol. 39, no. 10, pp. 1525-1531, Oct. 1991.

[8] A. Kashyap, T. Basar, and R. Srikant, "Correlated Jamming on MIMO Gaussian Fading Channels," IEEE Trans. Inf. Theory, vol. 50, no. 9 , pp. 2119-2123, Sept. 2004

[9] Y. E. Sagduyu and A. Ephremides, "SINR-Based MAC Games for Selfish and Malicious Users," Proc. Information Theory and Applications Workshop, San Diego, CA, Jan. 2007.

[10] Y. E. Sagduyu and A. Ephremides, "A Game-Theoretic Analysis of Denial of Service Attacks in Wireless Random Access," Proc. WiOpt07, Limassol, Cyprus, Apr. 2007.

[11] E. Altman, K. Avratchenkov, and A. Garnaev, "A Jamming Game in Wireless Networks with Transmission Cost," Proc. Net-Coop, Avignon, France, June 2007.

[12] Y. Liu, C. Comaniciu, and H. Man, "A Bayesian Game Approach for Intrusion Detection in Wireless Ad Hoc Networks," Proc. GameNets, Pisa, Italy, Oct. 2006.

[13] S. Radosavac, A. A. Cardenas, J. S. Baras, and G. V. Moustakides, "Detecting IEEE 802.11 MAC Layer Misbehavior in Ad Hoc Networks: Robust Strategies Against Individual and Colluding Attackers,' Journal of Computer Security, vol. 15, no. 1, pp. 103-128, Jan. 2007.

[14] G. Theodorakopoulos and J. Baras, "Game Theoretic Modeling of Malicious Users in Collaborative Networks," IEEE J. Sel. Areas Commun., vol. 26, no. 7, pp. 1317-1327, Sept. 2008.

[15] H. Inaltekin and S. B. Wicker, "Random Access Games: Selfish Nodes with Incomplete Information," Proc. IEEE MILCOM, Orlando, FL, Oct. 2007.

[16] Mingyan Li, I. Koutsopoulos, and R. Poovendran, "Optimal Jamming Attack and Network Defense Policies in Wireless Sensor Networks," Proc. IEEE INFOCOM, Anchorage, AK, May 2007.

[17] M. Babaioff, R. Kleinberg, and C. H. Papadimitriou, "Congestion Games with Malicious Players," Proc. ACM Conf. Electronic Commerce, San Diego, CA, June 2007. 